\title{
Délibérations du Comité central de la FMH
}

St. Lors de sa séance du 15 février 2001, le Comité central a traité, entre autres, des affaires suivantes:

\section{Réforme des structures, droit professionnel}

1. Réponse à la consultation sur les Ordonnances concernant les produits thérapeutiques

Le Comité central donne son aval à la prise de position de la FMH, invitée à se prononcer à cet égard. Il relève toutefois que la loi fédérale sur les produits thérapeutiques (LFP) est si détaillée qu'elle ne laisse à première vue que peu de latitude pour des modalités d'application dans les ordonnances et que les conséquences des nouvelles réglementations ne peuvent encore être évaluées à titre définitif. Cela étant, il se limite à quelques aspects qui lui paraissent importants. L'avenir et la pratique du nouvel institut montreront où il conviendra d'agir.

La réponse de la FMH peut être consultée sur internet (www.fmh.ch, bande de roulement de la page d'accueil).

\section{Assurances sociales}

1. Recommandations concernant le diagnostic mammaire par palpation (circ. 1/2001 B)

La FMH a pour objectif d'unifier et de promouvoir d'une manière générale, par des exigences de qualité définies, la valeur analytique et l'applicabilité des recommandations sur le plan suisse. S'étant fixée un résultat déterminé, elle n'est pas totalement neutre. Cela dit, la FMH, contrairement à d'autres organisations, se considère prédestinée à prendre en mains la coordination des recommandations supradisciplinaires en raison de ses contacts étroits et constants avec les sociétés de discipline médicale. Diverses publications dans le Bulletin des médecins suisses témoignent de cette volonté de promotion de la qualité. Les premières recommandations choisies sont, parmi d'autres thèmes, celles pour le diagnostic et le traitement du carcinome mammaire, parce que c'est un domaine complexe de la médecine qui évolue rapidement et qui concerne tant le médecin de premier recours que les spécialistes et superspécialistes et parce qu'il constitue un champ d'expérimentation approprié par rapport aux objectifs de la FMH. De plus, ce problème concerne presque la moitié de notre population, qui a droit de bénéficier des dernières connaissances en la matière et, dans la mesure du possible, d'examens et de traitements unifiés. Pour l'élaboration de ces recommandations - dont seule la partie concernant le diagnostic sera publiée pour le moment - dix sociétés de discipline médicale ont été invitées à déléguer au groupe de travail des spécialistes émanant si possible pour moitié du milieu hospitalier et pour l'autre de cabinets médicaux et représentant les différentes régions linguistiques. Le Comité central prend connaissance du rapport de la commission pour les recommandations et les approuve ainsi que l'introduction du Dr E. Eicher.

\section{Recommandations "Dépassement de terme»}

Le Comité central approuve la version présentée des Recommandations pour la surveillance et la prise en charge en cas de dépassement de terme, déjà adoptée par la commission.

\section{Prévention}

\section{Législation sur la stérilisation}

A la séance de juin 2000 du Sénat de l'ASSM, il a été décidé de ne pas mettre en vigueur les nouvelles directives concernant la stérilisation et d'attendre que les choses soient clarifiées en matière de législation. Le mandat a été simultanément confié à la sous-commission d'ajouter aux directives de 1981 une recommandation aux médecins de renoncer, en matière de prévention de la grossesse, aux stérilisations chirurgicales et d'opter en faveur des nombreuses méthodes réversibles.

Une rencontre a eu lieu en juillet 2000 avec la commission pour les questions juridiques du Conseil national qui devra déterminer s'il y a lieu d'élaborer une loi spéciale ou si la révision du droit sur les tutelles devait être ajournée.

Le Département fédéral de la justice a mis sur pied une sous-commission qui, dans le cadre de la révision de la loi sur les tutelles (à l'avenir «droit sur la protection des personnes») devrait élaborer une proposition conforme à la loi.

Le Comité central prend acte du rapport sur les travaux législatifs en matière de stérilisation.

\section{Formation postgraduée et continue}

1. Nouvelle systématique des titres de spécialiste Le Comité central approuve les documents tels qu'ils sont présentés à l'intention de la Chambre médicale.

Les principaux projets de révision de programmes peuvent être consultés sur internet (www.fmh.ch $\Rightarrow$ FP \& FC $\Rightarrow$ projets de programmes de formation postgraduée en révision). Propositions et remarques peuvent être adressées par e-mail au Département de la formation postgraduée et continue.

\section{Les accords bilatéraux avec l'UE et la loi} sur l'exercice des professions médicales La FMH règle depuis 70 ans la formation postgraduée en vue de l'obtention d'un titre de spécialiste. En tant qu'association privée, la FMH s'acquitte ainsi d'une tâche importante qui relève en fait du droit public. L'entrée en vigueur des accords bilatéraux et, dans la 
foulée, de la loi sur l'exercice des professions médicales (LEPM) et de l'ordonnance y afférente, mettra un terme, probablement au $1^{\mathrm{er}}$ janvier 2002, à l'autonomie de la FMH en matière de réglementation de la formation postgraduée et continue des médecins. A partir de cette date, la haute surveillance des études et de la formation postgraduée et continue sera assumée par la Confédération. Cependant, dans le cadre d'une accréditation de la Réglementation de la formation postgraduée (RFP) et des programmes de formation s'y rapportant, la FMH continuera à régler et organiser la formation postgraduée des médecins.

Principales innovations: A partir du $1^{\mathrm{er}}$ janvier 2002, plus aucun titre de spécialiste FMH ne sera délivré. Ils seront remplacés par des titres fédéraux postgrades, selon l'annexe de l'ordonnance de la loi sur l'exercice des professions médicales. Alors que jusqu'ici le détenteur d'un titre FMH devait obligatoirement s'affilier à la FMH (ce qui a contribué au niveau élevé d'organisation de la FMH), à l'avenir, le titulaire d'un titre fédéral postgrade n'y sera plus tenu. Pour cette raison, le système de financement actuel du domaine de la formation postgraduée et continue, alimenté principalement par les cotisations des membres, ne pourra plus être maintenu.

Après s'être penché attentivement sur le problème, le Comité central a formulé les principes suivants: le Département de la formation postgraduée et continue doit être géré en tant que centre de charges financièrement séparé des autres domaines de la FMH. Le financement de ce département sera couvert par les prestations fournies et éventuellement par une contribution spéciale encore à définir, émanant des cotisations générales. Toutes les dépenses du département doivent être transparentes et facturées à leur coût réel lors de l'octroi d'un titre de spécialiste ou pour d'autres prestations. Les médecins en formation membres de la FMH et qui désirent le rester doivent être récompensés pour leur affiliation en bénéficiant, lors de l'octroi du premier titre fédéral postgrade, d'une réduction de la cotisation de membre ou du remboursement d'une partie de celle-ci. Ces principes garantissent que les non-membres de la FMH s'acquittent des coûts effectifs liés à un titre de spécialiste et ne bénéficient pas d'un subventionnement par les membres. Simultanément, la FMH doit aussi rendre l'affiliation financièrement attrayante pour les candidats à un titre fédéral postgrade.

Le CC donne son aval au document préparé à l'intention de la Conférence des présidents et de la Chambre médicale.

L'aide-mémoire FMH/OFSP figurant sur la page d'accueil de la FMH (www.fmh.ch $\Rightarrow$ FP \& FC) donne une information exhaustive sur les accords bilatéraux, la loi sur l'exercice des professions médicales et la formation prégraduée, postgraduée et continue.

\section{Organisation}

\section{Affaire de l'Hôpital de Laufon}

Vers la fin de l'année dernière, une transaction judiciaire a mis fin au litige qui opposait le Gouvernement de Bâle-Campagne au Dr Arnold Kaiser, médecin-chef. La décision concernant son licenciement immédiat en janvier 1999 a été annulée et son contrat de travail a été résilié au 15 novembre 2000. Comme l'écrivait la «Neue Zürcher Zeitung», le Gouvernement cantonal et M. Eduard Belser, directeur des affaires sanitaires à l'époque, ne sortent pas grandis de cette affaire.

Le Comité central, qui ne peut que déplorer la manière adoptée par un gouvernement cantonal pour se séparer d'un médecin-chef, collaborateur de longue date et apprécié de la population, a pris acte avec satisfaction de l'issue de ce litige. L'annulation de la décision du licenciement de ce médecin-chef par les autorités judiciaires est une première peu glorieuse.

Deutsch erschienen in Nr. 13/2001 